\title{
Force chains in sheared granular media of irregular particles
}

\author{
Andres A. Peña*, Hans J. Herrmann ${ }^{\dagger}$ and Pedro G. Lind** \\ ${ }^{*}$ Bilfinger Berger GmbH, Gustav-Nachtigal 3, 65915 Wiesbaden, Germany; \\ ICP, University of Stuttgart, Pfaffenwaldring 27, D-70569 Stuttgart, Germany \\ ${ }^{\dagger}$ Departamento de Física, Universidade Federal do Ceará, 60451-970 Fortaleza, Ceará, Brazil; \\ Computational Physics, IfB, HIF E12, ETH Hönggerberg, CH-8093 Zürich, Switzerland \\ ${ }^{* *}$ CFTC, Complexo Interdisciplinar, Univ. Lisboa, Av. Prof. Gama Pinto 2, 1649-003 Lisboa, Portugal; \\ ICP, University of Stuttgart, Pfaffenwaldring 27, D-70569 Stuttgart, Germany
}

\begin{abstract}
We study topological and dynamical properties in sheared granular media particularly when avalanches occur, i.e. at the abrupt increase of the total kinetic energy during the shear process. Our model considers irregular polygonal particles compressed between two moving plates. At the occurrence of an avalanche, we monitor the time evolution of the underlying contact force network. When all contact forces are considered, topological and dynamical quantities vary as a reaction to the avalanche, while the decomposition of the contact force network into strong and weak contacts uncovers a topology that is strongly correlated with the occurrence of avalanches.
\end{abstract}

Keywords: Force chains, Shear systems, Granular media, Avalanches PACS: $83.80 . \mathrm{Fg}, 45.70 .-\mathrm{n}, 81.05 . \mathrm{Rm}, 05.40$. -a

\section{INTRODUCTION}

One decade ago Radjai and co-workers described for the first time the bimodal character of the force network in granular packings [1]. In short, the network of contact forces can be decomposed in two subnetworks, one weak network comprehending the majority of the particles, each one carrying less than the average load, and one strong network with a few particles having contact forces larger than the average load. Till recently, this picture has been explored in several situations such as jamming processes [2], dissipation [3], polydispersity [4], deformation [5] and shear systems [6]. In all these studies forces are transmitted through particle contacts, and such a network of contact forces experience the onset of anisotropy under geometrical anisotropy (e.g. shear). However, while the mechanism of such anisotropy in systems with regular shaped particles is known, the scenario for particles anisotropic themselves (polygons) is, up to our knowledge, not so well understood.

The aim of this paper is twofold. First we generalize the algorithm to find force chains in a system of discs [7] to suit the more general situation of particles of irregular shape. Second, we study the evolution of such force chains in a sheared system, and focus on the particular case when an avalanche takes place [8].

We consider a two dimensional model of convex polygons to represent grains of some sheared granular material with a constant shear rate $\dot{\gamma}$. The deformation of the grains is modelled by letting them overlaping [8]. The contact plane is defined from the intersection points of each two overlapping polygons and the normal contact

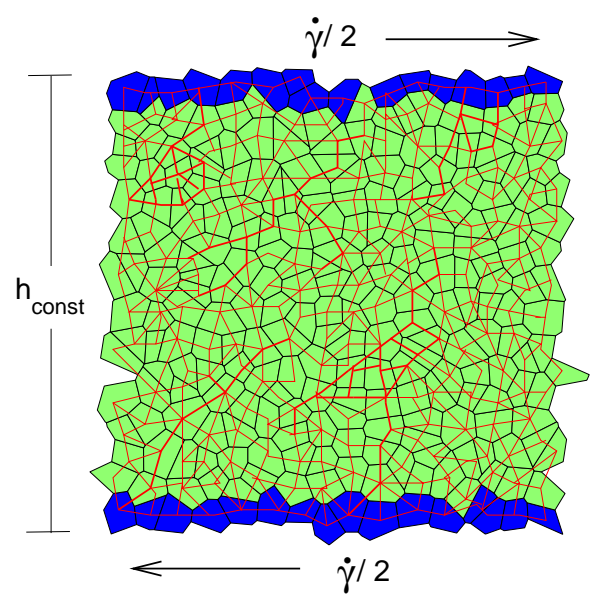

FIGURE 1. Sketch of the shear system of irregular polygonal particles (green) with the network of contact forces (red edges). Boundaries at constant shear rate $\dot{\gamma}$ are colored in blue.

force is perpendicular to the contact plane with a magnitude proportional to the overlap area. Connecting the centers of mass of touching particles yields the network of contact forces (Fig. 1).

\section{THE NETWORK OF CONTACTS}

We first consider the entire network of contact forces, Fig. 1, and study, at each time step, how the main topological properties [9] characterizing the network of con- 


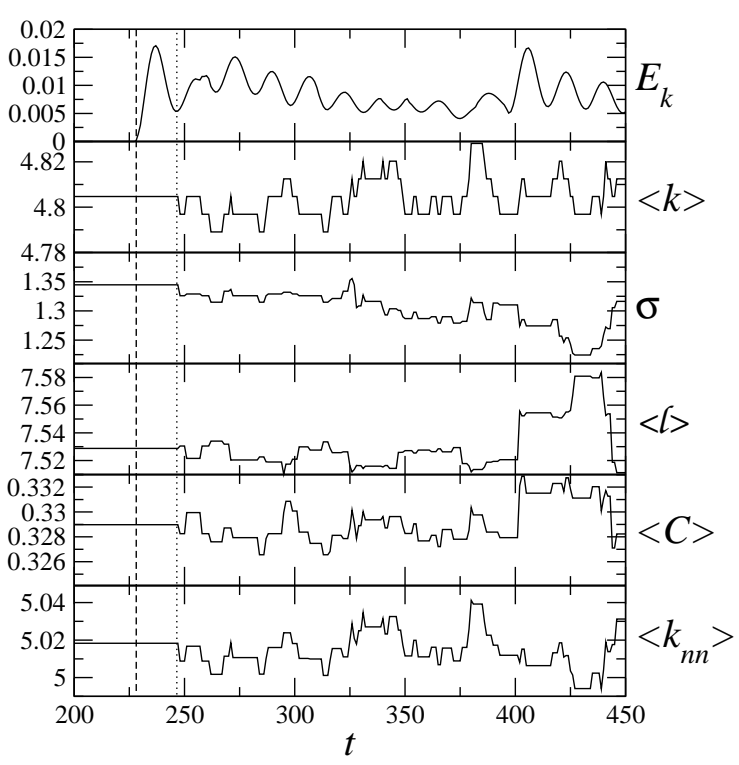

FIGURE 2. Monitoring network properties (see text) in a two-dimensional system of 256 irregular polygonal particles during an avalanche, which is marked by a sudden increase of the average kinetic energy $E_{k}$ per particle. The vertical dashed and dotted lines indicate the time-steps at which the avalanche occurs and the time-step at which the topology of the entire system starts to change.

tact forces evolve with the average kinetic energy

$$
E_{k}=\frac{1}{2 N} \sum_{i=1}^{N} m_{i} \vec{v}_{i} \cdot \vec{v}_{i}
$$

where $N$ is the number of particles and $m_{i}$ and $\vec{v}_{i}$ the mass and velocity of particle $i$ respectively. Figure 2 shows the evolution of $E_{k}$ together with the first two moments, $\langle k\rangle$ and $\sigma$, defining the coordination number distribution $P(k)$, the average shortest path length $\langle\ell\rangle$, the average clustering coefficient $C$ and the coordination correlation $k_{n n}$.

The average shortest path length $\langle\ell\rangle$ is defined as the average smallest number of contacts between two particles randomly chosen, the clustering coefficient $\langle C\rangle$ gives the average fraction of neighbouring particles that are touching each other, and the coordination correlation $k_{n n}(k)$ gives the average coordination number of the neighbors of one particle with coordination $k$.

From Fig. 2 one observes that all topological quantities start to vary in time at the same time step (vertical dotted line), as a response to the increase of the kinetic energy (vertical dashed line) marking the beginning of the avalanche [8]. Further, a closer look reveals that $\left\langle k_{n n}\right\rangle \simeq\langle k\rangle+0.2$ through time. From the direct inspection of these averages and with $k_{n n}=\int k^{\prime} P\left(k^{\prime} \mid k\right) d k^{\prime}$ one finds that $k_{n n}(k)=k$, as expected in such a system.

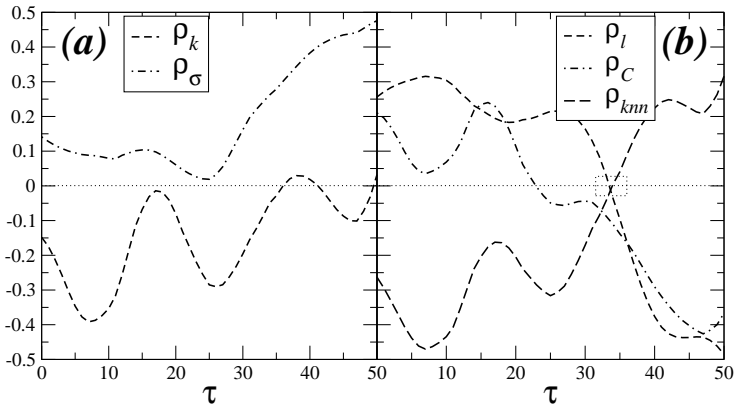

FIGURE 3. Correlation function between $E_{k}$, Eq. (2), and (a) the average coordination number $\langle k\rangle$ and the standard deviation $\sigma$ of $P(k)$ and also between (b) the three topological quantities $\langle\ell\rangle,\langle C\rangle$ and $\left\langle k_{n n}\right\rangle$ (see Fig. 1).

To see how the topological quantities are correlated with the kinetic energy we compute the correlation function of each one, namely

$$
\rho_{X}(\tau)=\frac{\left\langle(X(t+\tau)-\bar{X})\left(E_{k}(t)-\bar{E}_{k}\right)\right\rangle}{\sigma_{X} \sigma_{E_{k}}}
$$

where $X$ is one of the topological quantities described above and $\bar{X}$ symbolizes the time average of $X$ and $\sigma_{X}$ and $\sigma_{E_{k}}$ are the corresponding standard deviations. Figure 3 shows the correlation function of the five network properties as a function of the time-lag $\tau$.

In all cases, no large correlations are found, i.e. $\left|\rho_{X}\right|<$ 0.5 and the oscillations with length $\sim 18$ time-steps are due to the oscillating behavior of $E_{k}$ (see Fig. 2). For the two first moments of $P(k)$ (Fig. 3a) the correlation shows a tendency for increasing with the time-lag from medium negative to small correlation in the case of $\langle k\rangle$ and from small to medium positive correlation in the case of $\sigma$. The absence of oscillations in $\rho_{\sigma}$ is due to the continuous decrease of $\sigma$ as $E_{k}$ experiences a damping oscillation after the beginning of the avalanche.

In Fig. $3 b$ one sees behavior $\rho_{k n n}$ resembling the one of $\rho_{k}$, indicating a homogeneous evolution of the particle coordination with its vicinity. Curiously, both $\ell$ and $C$ show a decreasing correlation from medium positive to medium negative values. This indicates a first increase of these two properties at or just after the occurrence of the avalanche, and also an increase of $\ell$ and $C$ when $E_{k}$ decreases while the system is damping the released energy after the avalanche. The co-evolution of $\ell$ together with $C$ evidences a nontrivial behavior where the avalanche produces more connected local vicinities but larger paths connecting particles distant apart. Such feature can be understood by assuming that the avalanche produces separated force chains connecting the two shearing boundaries as observed in Ref. [7]. 
TABLE 1. Average values of the topological quantities for the weak and strong networks of contacts before and after the occurrence of avalanches (see text).

\begin{tabular}{ccccccc}
\hline & \multicolumn{2}{c}{ WEAK } & \multicolumn{2}{c}{ STRONG } & \multicolumn{2}{c}{ TOTAL } \\
\hline & Bef. & Aft. & Bef. & Aft. & Bef. & Aft. \\
\hline$\langle k\rangle$ & 3.16 & $3.12 \pm 0.1$ & 2.44 & $2.50 \pm 0.07$ & 4.80 & $4.81 \pm 0.01$ \\
$\sigma$ & 1.55 & $1.56 \pm 0.11$ & 1.12 & $1.11 \pm 0.09$ & 1.34 & $1.30 \pm 0.03$ \\
$\langle\ell\rangle$ & 8.75 & $9.58 \pm 0.80$ & 3.81 & $4.72 \pm 0.99$ & 7.53 & $7.53 \pm 0.02$ \\
$\langle C\rangle$ & 0.27 & $0.28 \pm 0.01$ & 0.27 & $0.29 \pm 0.02$ & 0.33 & $0.333 \pm 0.001$ \\
$\left\langle k_{n n}\right\rangle$ & 3.50 & $3.46 \pm 0.10$ & 2.72 & $2.79 \pm 0.07$ & 5.02 & $5.02 \pm 0.01$ \\
$\lambda$ & -1.35 & $-1.34 \pm 0.01$ & -0.66 & $-0.67 \pm 0.01$ & -1.02 & $-1.017 \pm 0.006$ \\
$\frac{\lambda}{10-4} \theta$ & -0.99 & $-1.06 \pm 0.10$ & -1.01 & $-1.10 \pm 0.11$ & -1.00 & $-1.10 \pm 0.11$ \\
\hline
\end{tabular}

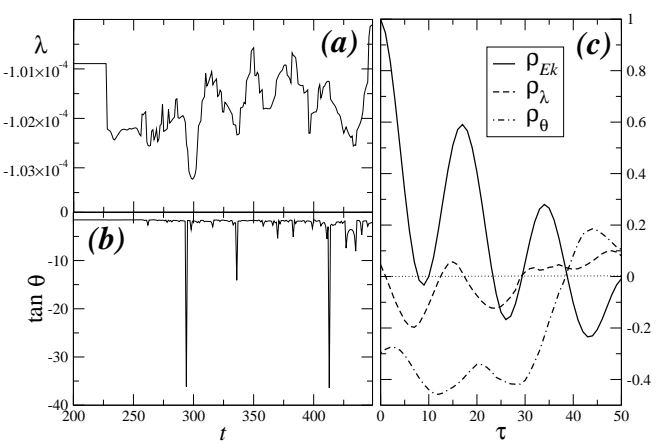

FIGURE 4. Time evolution of (a) the minor principal stress $\lambda_{-}$, Eq. (4) and (b) the direction $\tan \theta$ of the corresponding eigenvector (see text). In (c) the correlation functions for $\lambda$ and $\theta$ are plotted together with the autocorrelation function for $E_{k}$.

The stress tensor of the particles $\varepsilon$ can be defined as

$$
\varepsilon_{i j}=\frac{1}{V} \sum_{n=1}^{N_{c}} F_{i} r_{j}
$$

where $V$ is the particle volume, $N_{c}$ is the number of contacts of the particle, $F_{i}$ is the contact force and the $r_{j}$ the branch vector. We further assume density $\rho=$ 1 , yielding $m=V$. For irregular polygons the stress tensor is not symmetric since the contact force and the branch vector are in general not co-linear. Therefore, the principal stresses (eigenvalues) are

$$
\lambda_{ \pm}=\frac{\varepsilon_{11}+\varepsilon_{22}}{2} \pm \sqrt{\left(\frac{\varepsilon_{11}-\varepsilon_{22}}{2}\right)^{2}+\varepsilon_{12} \varepsilon_{21}}
$$

Since the Discrete Element Method we use adopts a tension-positive convention, the minor principal stress is $\lambda_{-}$and corresponds to the direction

$$
\tan \theta=\frac{\varepsilon_{11}-\varepsilon_{22}}{2 \varepsilon_{21}}-\sqrt{\left(\frac{\varepsilon_{11}-\varepsilon_{22}}{2 \varepsilon_{21}}\right)^{2}+\frac{\varepsilon_{12}}{\varepsilon_{21}}} .
$$

Figures $4 \mathrm{a}$ and $4 \mathrm{~b}$ shows the evolution of both $\lambda_{-}$ and $\tan \theta$, respectively, together with their correlation functions and the autocorrelation $\rho_{E k}$. Differently from the network properties, the minor stress experiences an abrupt decrease precisely when the avalanche occurs as the energy stored at the contacts is released, and it maintains an anti-correlation with $E_{k}$ during two oscillations. For the direction of the largest compression remains approximately constant with abrupt single variations corresponding to rearrangements of the particles inside the system. In Tab. 1 one further sees that the preferred direction makes an angle of $3 \pi / 4$ with the direction of shearing at the boundaries.

Looking the two rightmost columns in Tab. 1 where a comparison of all the above properties are given, before and after the avalanche, we can conclude that all quantities remain on average at the same values before and after the avalanche, within numerical errors, except the standard deviation $\sigma$ of $P(k)$ and the clustering coefficient.

\section{WEAK AND STRONG FORCES}

For the weak and strong contact force we use the criterion described in Ref. [7] i.e. we consider one particle to belong to the strong contact network if the smallest eigenvalue of its stress tensor is larger than the average value from the sample of all $N$ particles. Otherwise it belongs to the weak network.

From Tab. 1 one sees that $\langle k\rangle$ of the weak network is larger than the one for the strong network and both are smaller than the overall $\langle k\rangle$, due to the existence of separated force chains where many contacts connect particles in the strong network with particles in the weak network. This structure in parallel force chains is also responsible for a clustering coefficient and coordination correlation of the subnetworks smaller than the ones for the entire system. For $\sigma$ one finds a smaller value for the strong network, indicating a more localized $P(k)$, the entire network having an intermediate value. The average shortest path length is smaller for the strong network, indicating a small size of its composing chains. As for

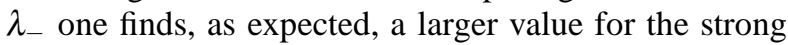
network followed by the value for the entire network. 


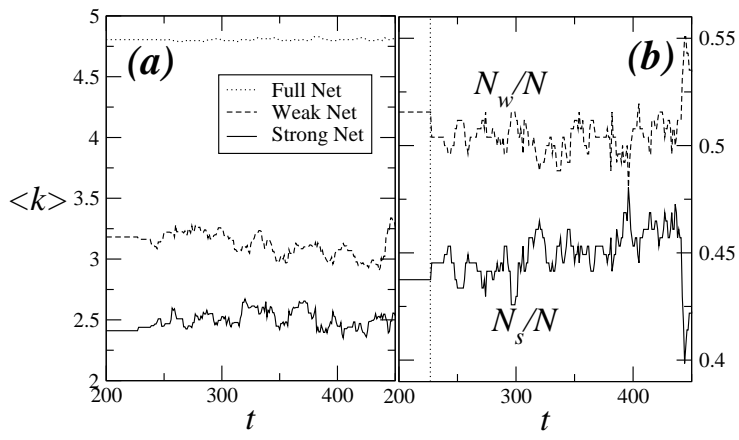

FIGURE 5. (a) Comparison of the average number $\langle k\rangle$ of contacts for the full network of contacts with the one for the weak and strong networks separately. (b) Time evolution of the number of contacts $N_{w}$ and $N_{s}$ for the weak and for the strong networks respectively.

The direction of the minor principal stress is, as stated above, almost $3 \pi / 4$.

Still from Tab. 1 one observes that, while for the entire network only $\sigma$ and $\langle C\rangle$ remain the same before and after the avalanche, for the two subnetworks, the properties that change after an avalanche are precisely the other three properties. Differently from the entire network (see Fig. 2), for the subnetworks these start to fluctuate precisely at the occurrence of the avalanche. Figure 5a illustrates this feature for the average coordination. Further, after an avalanche, the fluctuations of all properties are typically larger for each subnetwork when compared with the full network. Figure $5 \mathrm{~b}$ shows the fraction of particles at each subnetwork, with the vertical dotted line indicating the occurrence of the avalanche.

Finally, while the kinetic energy of the weak network evolves similarly to the full kinetic energy, for the strong network one observes a delay for the beginning of the fluctuation (Fig. 6a) and a shift in the correlation function (Fig. 6b).

\section{DISCUSSION AND CONCLUSIONS}

We showed how to extract some main features during avalanches in shear systems by measuring the topological properties of the underlying network and applied the criterion of bimodal contact force network to a situation where the particles composing the system are irregular polygons. By looking to the topology of the weak and strong subnetworks we described how the underlying structure may be infered. Namely, the combined results of the average shortest path length and correlation coordination of the subnetworks together with the entire system shows the occurrence of strong force chains interweaved with regions of weak forces.

Further, we showed that these subnetworks are good

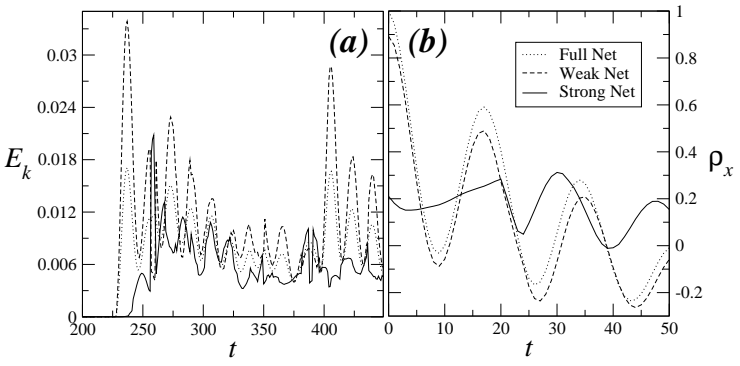

FIGURE 6. (a) Comparison of the kinetic energy $E_{k}$ for the full network of contacts with the one for the weak and strong networks and (b) their corresponding correlation functions as defined in Eq. (2).

decompositions to obtain better avalanches predictors, even when polygonal irregular particles are considered. Namely, an avalanche typically starts when either $\sigma$ or $\langle C\rangle$ change within the weak and strong subnetworks.

Forthcoming studies could be based on our findings. For instance, the application of Bayes theorem to ascertain up to which point is it possible to predict an avalanche from the structure of the network of contact forces, as well as the evaluation of the network of sliding contacts. Finally, the clustering coefficient and coordination correlation could be combined to ascertain how branched are the chains of forces in the strong network.

\section{ACKNOWLEDGMENTS}

The authors thank the support by German-Israeli Foundation and by Deutsche Forschungsgemeinschaft, under the project HE2732781. PGL thanks support by Deutsche Forschungsgemeinschaft, under the project LI 1599/1-1. HJH thanks the Max Planck prize.

\section{REFERENCES}

1. F. Radjaim D.E. Wolf, M. Jean and J.-J. Moreau, Phys. Rev. Lett. 80, 61 (1998).

2. M.E. Cates, J.P. Wittmer, J.P. Bouchaud and P. Claudin, Phys. Rev. Lett. 81, 1841 (1998).

3. W.J. Wang, Z.G. Zhu, Europhys. Lett. 82, 24004 (2008).

4. M. Muthuswamy and A. Tordesillas, J. Stat. Mech. $\mathbf{P 0 9 0 0 3}$ (2006).

5. N.P. Kruyt and S.J. Antony, Phys. Rev. E 75, 951308 (2007).

6. S.D.C. Walsh, A. Tordesillas, J.F. Peters, Gran. Matt. 9, 337 (2007)

7. J.F. Petersm M. Muthuswamy, J. Wibowo and A. Tordesillas, Phys. Rev. E 72, 041307 (2005).

8. A.A. Peña, S. McNamara, P.G. Lind and H.J. Herrmann, Granular Matter, accepted (2008).

9. S. Boccaletti, V. Latora, Y. Moreno, M. Chavez and D.-U. Hwanga, Phys. Rep. 424, 175-308 (2006). 